\title{
Some Results of The Coprime Graph of a Generalized Quaternion Group $Q_{4 n}$
}

\author{
Nurhabibah ${ }^{1}$, Abdul Gazir Syarifudin ${ }^{2}$ and I Gede Adithya Wisnu Wardhana ${ }^{3 *}$ \\ 1,2,3 Department of Mathematics, Universitas Mataram \\ Email: habibahmtk05@gmail.com, abdgazirsyazir@gmail.com, *adhitya.wardhana@unram.ac.id \\ *correspondence author
}

\begin{abstract}
The Coprime graph is a graph from a finite group that is defined based on the order of each element of the group. In this research, we determine the coprime graph of generalized quaternion group $Q_{4 n}$ and its properties. The method used is to study literature and analyze by finding patterns based on some examples. The first result of this research is the form of the coprime graph of a generalized quaternion group $Q_{4 n}$ when $n=2^{k}, n$ an odd prime number, $n$ an odd composite number, and $n$ an even composite number. The next result is that the total of a cycle contained in the coprime graph of a generalized quaternion group $Q_{4 n}$ and cycle multiplicity when $n$ is an odd prime number is $n-1$.
\end{abstract}

Keywords: coprime graph; generalized quaternion group; order, path.

\begin{abstract}
Abstrak
Graf koprima merupakan graf dari dari suatu grup bingga yang didefiniskan berdasarkan orde dari masing-masing elemen grup tersebut. Pada penelitian ini akan dibabas tentang bentuk graf koprima dari grup generalized quaternion $Q_{4 n}$. Metode yang digunakan dalam penelitian ini adalah studi literatur dan melakukan analisis berdasarkan pola yang ditemukan dalam beberapa contoh. Adapun basil pertama dari penelitian adalab bentuk graf koprima dari grup generalized quaternion $Q_{4 n}$ untuk kasus $n=2^{k}, n$ bilangan prima ganjil ganjil, $n$ bilangan komposit ganjil dan $n$ bilangan komposit genap. Hasil selanjutnya adalah total sikel pada graf koprima dari grup generalized quaternion dan multiplisitas sikel ketika $n$ bilangan prima ganjil adalah $n-1$.

Kata kunci: graf koprima; grup generalized quternion; orde; lintasan.
\end{abstract}

\section{INTRODUCTION}

A graph $G$ consists of a non-empty finite set $V(G)$ of elements called vertices and a finite family of $E(G)$ of unordered pairs of (not necessarily distinct) elements of $V(G)$ called edges [1]. Some graph representations of a group are identity graph, inverse graph, commuting, non-commuting graph, coprime graph, non-coprime graph, and others. The coprime graph is a graph that represents a finite group where vertices are all elements of that group, and two distinct vertices are adjacent if and only if its order pairwise relative prime.

In recent years, there are some researches about the representation graph of a group. In 2010, Vahidi and Talebi [2] researched the properties of the commuting graph of the dihedral group and quaternion group and got some parameters of graph theory. In 2017, Abdussakir [3] researched the commuting graph of a dihedral group that is matrix dimension, cycle multiplicity, radius, and diameter. In the next year, Syarifuddin et al. [4]. Researched some characterizations of a dihedral group's coprime graph got the form of graph, radius, diameter, and girth.

Submitted February 1 $1^{\text {th }}, 2021$, Revised March 1st 2021 , Accepted for publication March $1^{\text {st }}, 2021$.

This is an open access article under CC-BY-SA license (https://creativecommons.org/licence/by-sa/4.0/) 
From that, this research will present the coprime graph of a generalized quaternion group $Q_{4 n}$ and its properties, such as the form of a graph, total of cycles, and cycle multiplicity.

\section{METHOD}

This research aims to study literature studying generalized quaternion group, the order of elements a group, coprime graph, and its properties from some references. After that, the authors construct and analyze the coprime graph of the generalized quaternion group $Q_{4 n}$ by dividing, the case of $n$, construct the conjecture based on the pattern in examples and prove it. If the conjecture is untrue, then the author will construct another conjecture and prove it, but if the conjecture is true, then it is stated as a theorem.

\section{RESULTS AND DISCUSSION}

The authors will discuss the form of the coprime graph of the generalized quaternion group $Q_{4 n}$, a total of cycles contained in a graph and its cycle multiplicity.

\subsection{Coprime Graph of $Q_{4 n}$}

Generalized quaternion group $Q_{4 n}$ is define as follows.

Definition 1. ( [5] The generalized quaternion group $Q_{4 n}(n \geq 2)$ is a group with a presentation of the form

$$
<a, b \mid a^{2 n}=e, a^{n}=b^{2}, b^{-1} a b=a^{-1}>.
$$

It is easy to see that $Q_{4 n}$ is of order $4 n, a$ has order $2 n, b$ has order four, and the relation $a^{k} b=b a^{-k}$ holds for all $k \in \mathbb{Z}[6]$.

The generalized quaternion group will be represented as the coprime graph, which is defined as follows.

Definition 2. ( [6]) Let $G$ be finite group, the coprime graph of $G$ denoted by $\Gamma_{G}$ is a graph with vertices are elements of $\mathrm{G}$ and two distinct vertices $x$ and $y$ are adjacent if and only if $(|x|,|y|)=1$.

In this research, will be determined the form of the coprime graph of the generalized quaternion group $Q_{4 n}$. The results of this research show that there are four forms of the coprime graph of the generalized quaternion group $Q_{4 n}$ based on $n$. Theorem 1 explains the form of a coprime graph of the generalized quaternion group $Q_{4 n}$ for $n=2^{k}$ where $k$ is a natural number.

Theorem 1. Let $Q_{4 n}$ be a generalized quaternion group. If $n=2^{k}$ then the coprime graph of $Q_{4 n}$ is complete bipartite.

Proof. Let $Q_{4 n}=\left\{e, a, a^{2}, \ldots, a^{2 n-1}, b, a b, a^{2} b, \ldots, a^{2 n-1} b\right\}$. Let $Q_{4 n}$ partitioned into two sets $P_{1}=$ $\{e\}$ and $P_{2}=Q_{4 n} \backslash\{e\}$. For all $x, y \in P_{2}$, the order of $x$, and $y$ are $2^{i}$ and $2^{j}$ for $i, j \in\{1, \ldots, k+1\}$. Then 2 divides $(|x| .|y|)$, hence $x$ and $y$ are not adjacent and $\Gamma_{Q_{4 n}}$ is a bipartite graph. Since $|e|=1$ and $|z|=2^{m}$ for $m \in\{1, \ldots, k+1\}$ for any $y \in P_{2}$, we have $(|e|,|y|)=1$. Hence $e$ is adjacent with all vertices in $P_{2}$. Thus the coprime graph of $Q_{4 n}$ with $n=2^{k}$ is complete bipartite. 
The form of the coprime graph of the generalized quaternion group $Q_{4 n}$ when $n$ is an odd prime number will be presented in the following theorem.

Theorem 2. Let $Q_{4 n}$ generalized quaternion group. If $n=p, p$ is an odd prime number, then the coprime graph of $Q_{4 n}$ is tripartite.

Proof. Let $Q_{4 n}=\left\{e, a, a^{2}, \ldots, a^{2 p-1}, b, a b, a^{2} b, \ldots, a^{2 p-1} b\right\}$, so the order of each element is $|e|=1$, $\left|a^{p}\right|=2,\left|a^{i} b\right|=4$ for each $i \in\{0,1,2, \ldots, 2 p-1\},\left|a^{2 j}\right|=p$ for each $j \in\{1,2, \ldots, p-1\}$, and $\left|a^{k}\right|=$ $2 p$ for each $k \in\{1,3, \ldots, p-2, p+2, \ldots, 2 p-1\}$. So the order of each $x \in Q_{4 n}$ is $1,2, p, 4$, or $2 p$. Since $p \neq 2$, we can define three partitions of $Q_{4 n}$ such that the order of each element in the same partition is not pointwise relative prime. Those partitions are $P_{1}=\left\{x \in Q_{4 n}|| x \mid=1\right\}=\{e\}, \quad P_{2}=$ $\left\{x \in Q_{4 n} \| x \mid=2 l, l \in \mathbb{N}\right\}$ and $P_{3}=\left\{x \in Q_{4 n} \| x \mid=p\right\}$. It is easy to see that every pair $x, y \in P_{i}$ are not adjacent since $(|x|,|y|) \neq 1$, thus the coprime graph of $Q_{4 n}$ when $n$ is an odd prime number is tripartite.

Note that the coprime graph of $Q_{4 n}$ in Theorem 2 cannot be complete tripartite since the order of $a$ is always $2 n$. The next theorem explains the coprime graph's form of the generalized quaternion group $Q_{4 n}$ for every composite number $n$.

Theorem 3. Let $Q_{4 n}$ be a generalized quaternion group. If $n=p_{1}^{k_{1}} p_{2}^{k_{2}} \ldots p_{m}^{k_{m}}, p_{1}=2, p_{i}$ are distinct prime number then the coprime graph of $Q_{4 n}$ is $m+1$ partite.

Proof. First, we can assume that $p_{i}<p_{j}$ whenever $i<j$, then we define $m+1$ subset of $Q_{4 n}$. The first subset is the set of the element with order $n_{1}=1$ or, precisely, $P_{1}=\{e\}$. For $j=2, \ldots, m+1$, we define the partition $P_{j}$, is the set of the element with order $n_{j}$ where $p_{j-1} \mid n_{j}$ but $p_{s} \nmid p_{j}$ for each $s<j$. By this definition, the $m+1$ subsets form a partition in $Q_{4 n}$. For $j>0$, the order of elements from $P_{j}$ is not pairwise relative prime, or its order can be divided by $p_{j}$. Hence the coprime graph of $Q_{4 n}$ is $m+1$ partite.

And for $n$ is an odd composite number, then we have a different form of the coprime graph of the generalized quaternion group $Q_{4 n}$.

Theorem 4. Let $Q_{4 n}$ generalized quaternion group. If $n=p_{1}^{k_{1}} p_{2}^{k_{2}} \ldots p_{m}^{k_{m}}, p_{i} \neq 2, p_{i}$ are distinct prime number then the coprime graph if $Q_{4 n}$ is $m+2$ partite.

Proof. First, we can assume that $p_{i}<p_{j}$ whenever $i<j$, then we define $m+2$ subset of $Q_{4 n}$. The first subset is the set of the element with order $n_{0}=1$ or, to be exact, $P_{0}=\{e\}$. The second partition is $P_{1}$, the set of the element with order $n_{1}$, where $2 \mid n_{1}$. The third partition is $P_{2}$, the set of the element with order $n_{2}$, where $p_{1} \mid n_{2}$. For $j=3, \ldots, m+2$, we define the partition $P_{j}$, is the set of the element with order $n_{j}$ where $p_{j-1} \mid n_{j}$ but $p_{s} \nmid p_{j}$ for each $s<j$. By this definition, the $m+2$ subset forms a partition in $Q_{4 n}$. For $j>1$, the order of elements from $P_{j}$ are not pairwise relative prime, or its order can be divided by $p_{j}$. And the order of any elements of $P_{1}$ is always divided by two. Hence the coprime graph of $Q_{4 n}$ is $m+2$ partite.

\subsection{Total of Cycle and Cycle Multiplicity}

The definition of a cycle of a graph is given in the following definition.

Definition 3. ( [7]) Cycle is not a trivial closed path with each vertex is distinct. 
In this research, the author got a total of cycles contained in $\Gamma_{Q_{4 n}}$ when $n$ is an odd prime number, as explained in Theorem 5 as follows.

Theorem 5. Let $\Gamma_{Q_{4 n}}$ be the coprime graph of the generalized quaternion group $Q_{4 n}$. If $n$ is an odd prime number, then $\Gamma_{Q_{4 n}}$ has $2 n^{2}-n-1$ cycles.

Proof. Based on theorem 2, $\Gamma_{Q_{4 n}}$ is tripartite with partition are $P_{1}, P_{2}$, and $P_{3}$. The form of the cycle in this graph is $e-u-v-e$ with $u \in P_{2}$ and $v \in P_{3}$. From proof of theorem 2, the total elements in $P_{2}$ is $2 n+1$, and the total of elements in $P_{3}$ is $n-1$. So, there are possibilities $2 n+1$ vertices $u$ and $n-1$ vertices $v$. Based on multiplication rules, the total of cycles with form $e-u-v-e$ that is contained in $\Gamma_{Q_{4 n}}$ is $(2 n+1)(n-1)=2 n^{2}-n-1$. Thus $\Gamma_{Q_{4 n}}$ has $2 n^{2}-n-1$ cycles.

Based on Theorem 5 we now can find cycle multiplicity of $\Gamma_{Q_{4 n}}$ for $n$ is an odd prime. The definition of cycle multiplicity is defined as follows.

Definition 4. ([8]) Let $G$ is a graph, $V(G)$ and $E(G)$ is set of vertices and set of edges. $C M(G)$ is notation of cycle multiplicity defined by the maximum number of line disjoint cycles contained in $G$.

The last theorem explains the cycle multiplicity.

Theorem 6. Let $\Gamma_{Q_{4 n}}$ be the coprime graph of the generalized quaternion group $Q_{4 n}$. If $n$ is an odd prime number, then $C M\left(\Gamma_{Q_{4 n}}\right)=n-1$.

Proof. Let $V_{1}=\left\{u \in Q_{4 n}|| u \mid=4\right\}$ and $V_{2}=\left\{v \in Q_{4 n}|| v \mid=p\right\}$, so $e-u-v-e$ is a cycle for each $u \in V_{1}$ and $v \in V_{2}$. And then, let $e-u_{1}-v_{1}-e$ and $e-u_{2}-v_{2}-e$ is two distinct cycles in $\Gamma_{Q_{4 n}}$. Both cycles will adjoin edges if and only if $u_{1} \neq v_{1} \neq u_{2} \neq v_{2}$. Thus, the maximum number of line disjoint cycle is in $\Gamma_{Q_{4 n}}$ or $C M\left(\Gamma_{Q_{4 n}}\right)=\min \left\{\left|V_{1}\right|,\left|V_{2}\right|\right\}=\min \{2 n+1, n-1\}=n-1$.

\section{CONCLUSIONS}

The obtained results show that the coprime graph of the generalized quaternion group $Q_{4 n}$ when $n=2^{k}, n$ is an odd prime number, $n=p_{1}^{k_{1}} p_{2}^{k_{2}} \ldots p_{m}^{k_{m}}$ with $p_{1}=2, n=p_{1}^{k_{1}} p_{2}^{k_{2}} \ldots p_{m}^{k_{m}}$ with $p_{i} \neq 2$ is complete bipartite, tripartite, $m+1$ partite and $m+2$ partite, total of cycles contained in $\Gamma_{Q_{4 n}}$ when $n$ is an odd prime number is $2 n^{2}-n-1$ with $C M\left(\Gamma_{Q_{4 n}}\right)=n-1$.

\section{REFERENCES}

[1] R. J.Wilson, Introduction to Graph Theory, England: Pearson Education Limited, 2010.

[2] J. Vahidi and A. A. Talebi, "The commuting graph on groups $D_{2 n}$ and $Q_{n}$," The Journal of Mathematics and Computer Sciences, vol. 1, no. 2, pp. 123-127, 2010.

[3] Abdussakir, "Radius, Diameter, Multiplisitas Sikel, dan Dimensi Metrik Graf Commuting dari Grup Dihedral," JURNAL MATEMATIKA "MANTIK", vol. 03, no. 01, pp. 1-4, 2017.

[4] A. G. Syarifuddin, Nurhabibah, D. P. Malik and I. G. A. W. Wardhana, "Some Characterizations of Coprime Graph of Dihedral group D2n," Journal of Physics: Conference Series, vol. 1722, no. 1, pp. 1-4, 2021.

[5] B. Ahmadi and H. Doostie, "On the Periods of 2-Step General Fibonacci Sequences in the Generalized Quaternion Groups,” Discrete Dynamics in Nature Society, vol. 2012, 2012. 
[6] X. L. M. H. Q. Wei and L. Y. Yang, "The coprime graph of a group," International Journal of Group Theory, vol. 3, no. 3, pp. 13-23, 2014.

[7] Abdussakir, N. N. Azizah and Nofandika, Teori Graf, Malang: UIN Malang Press, 2009.

[8] G. Chartrand, D. Geller and S. Hedetniemi "Grpah with Forbidden Subgraphs," Journal of Combinatorial Theory Series B, vol. 10, no. 1, pp. 12-41, 1971. 